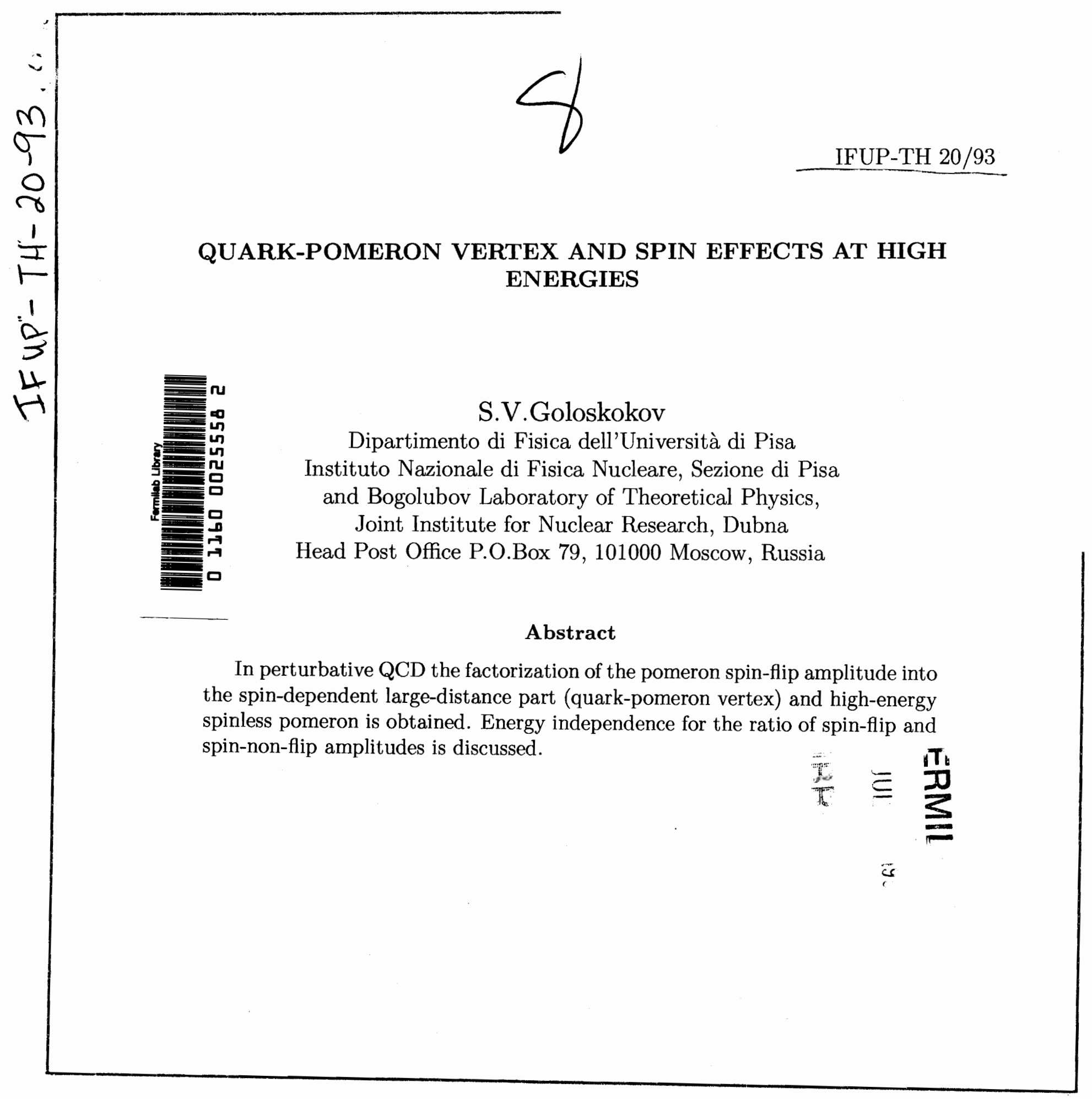


IFUP-TH $20 / 93$

\title{
QUARK-POMERON VERTEX AND SPIN EFFECTS AT HIGH ENERGIES
}

\author{
S.V.Goloskokov \\ Dipartimento di Fisica dell'Università di Pisa \\ Instituto Nazionale di Fisica Nucleare, Sezione di Pisa \\ and Bogolubov Laboratory of Theoretical Physics, \\ Joint Institute for Nuclear Research, Dubna \\ Head Post Office P.O.Box 79, 101000 Moscow, Russia ${ }^{1,2}$
}

\begin{abstract}
In perturbative QCD the factorization of the pomeron spin-flip amplitude into the spin-dependent large-distance part (quark-pomeron vertex) and high-energy spinless pomeron is obtained. Energy independence for the ratio of spin-flip and spin-non-flip amplitudes is discussed.
\end{abstract}

1 Permanent address

2 E-mail: goloskkv@theor.jinrc.dubna.su 
It is well known that in different high-energy reactions large spin effects are observed [1]. The standard perturbative QCD calculations cannot explain such experimentally observable phenomena in hard and soft reactions [2]. Note that there are many spin experimental data at high energies and fixed momenta transfer. In this kinematic region the exchange with vacuum quantum numbers in the $t$ - channel (pomeron) plays an important role. So the investigation of the pomeron spin structure is very important.

The vacuum $t$-channel amplitude is usually associated in QCD with the two-gluon exchange [3]. The nonperturbative properties of QCD which are important in the investigated region have been taken into account in some models for the spinless pomeron $[4,5]$. The spin structure of pomeron exchange has been analysed within a similar model in [6]-[8]. It was shown that different contributions like a gluon ladder [6] and quark loops [7] lead to the spin-flip amplitude growing as $s$ in the limit $s \rightarrow \infty$. As a result, the spin-flip amplitudes are suppressed only logarithmically with respect to the spin-non-flip amplitude

$$
\frac{\left|T_{\text {flip }}\right|}{\left|T_{\text {non-flip }}\right|} \simeq \frac{m \sqrt{|t|}}{a(m, t) \ln s / s_{0}} .
$$

Here $m=.33 \mathrm{GeV}$ is the constituent quark mass and $a$ is a function linearly dependent on $|t|$ at large $|t|$. This result confirms the absence of the spin-flip amplitudes only in the leading $\log$ approximation.

It is known that the quark-quark scattering plays an important role in different high energy processes. Previously, spin effects in this high-energy subprocess have been studied in the soft and semi-hard momentum transfer region. It was shown in ref. [6] within the qualitative QCD analysis that the $q q$ spin-flip amplitude growing as $s$ can be obtained in the $\alpha_{s}^{3}$ order. In ref. [8], the quantitative calculations of the spin effects in quark-quark scattering has been performed in the $\alpha_{s}^{3}$ order in the region $s \rightarrow \infty,|t|>1 \mathrm{GeV}^{2}$ where the perturbative theory can be used.

In the present paper, the factorization of the $q q$ spin-flip amplitude into the spindependent large-distance part and the high-energy spinless pomeron will be shown. This permits us to define the quark-pomeron vertex and to discuss the results of summation of the pomeron ladder graphs in higher orders of QCD. It will be shown that the obtained amplitude leads to the ratio of spin-flip and non-flip amplitudes independent of the energy in contrast with (1).

Let us investigate spin effects in the quark-quark scattering as $s \rightarrow \infty,|t|$-fixed in the perturbative QCD. We use the following definitions of the initial quark momenta:

$$
p_{1}=p+r, \quad p_{2}=p^{\prime}-r .
$$

In the investigated region

$$
s \simeq 2\left(p p^{\prime}\right), t=\frac{r^{2}}{4}=-\Delta^{2},(p r)=\left(p^{\prime} r\right)=0 .
$$


Diagrams, Fig.1, determine the born two-gluon contribution and amplitudes with the spin-flip in the upper quark line [6]. Note that there are radiative graphs (see [9] e.g.) but they do not contribute to the quark-quark spin-flip amplitude [8]. It can be shown that for the color singlet exchange the real parts are compensated in the sum of diagrams, Fig.1, and the corresponding $s \rightarrow u$ crossing graphs with crossed gluon lines. So we can calculate only the imaginary parts of diagrams in the case of $t$-channel pomeron exchange.

In what follows we shall analyse the amplitudes with the spin-non-flip matrix element in the down quark line. So we are interested in the spin effects only for the upper quarks, for simplicity. The imaginary parts of these matrix elements for the diagrams, Fig.1(a)-(b), can be written as follows (the light-cone variables [7] are used here):

$$
\begin{array}{r}
\operatorname{Im}\left\langle T_{i}(s, t)\right\rangle=c_{i} \frac{\alpha_{s}^{3}}{(2 \pi)^{2} s} \int_{s_{0} / s}^{1} \frac{d x}{x(1-x)} \int d^{2} k_{\perp} d^{2} l_{\perp} \\
\hat{N}\left(k_{\perp}, l_{\perp}, r, x\right)\left[\left(G_{i}^{1} G_{i}^{2}\right) \prod(F(l \pm r))\right],
\end{array}
$$

where $c_{i}$ is a color factor, $G_{i}^{1} G_{i}^{2}$ are the corresponding functions of $t$-channel propagators in the up part of the graph, $F(l \pm r)$ are the gluon propagators from the down part of the graph, $\hat{N}\left(k_{\perp}, l_{\perp}, r, x\right)$ is the matrix structure in the upper quark line matched with the gluon block. The spin-flip matrix elements of the function $\hat{N}\left(k_{\perp}, l_{\perp}, r, x\right)$ for diagrams, Fig.1a-c, have been calculated for the first time in [6]. It was shown that they are growing $\sim s^{2}$ as $s \rightarrow \infty$.

For diagram, Fig.1a, the functions $G$ and $F$ look as follows:

$$
\begin{gathered}
G_{a}^{1,2}=-\frac{1-x}{x^{2} m^{2}+(1-x) \lambda^{2}+\left[k_{\perp} \pm(1-x) r_{\perp}\right]^{2}} \\
F(l \pm r)=-\frac{1}{\lambda^{2}+\left[l_{\perp} \pm r_{\perp}\right]^{2}}
\end{gathered}
$$

where we introduce the mass $\lambda$ into the gluon propagators. It follows from (3) that in the $\lambda \rightarrow 0$ limit we have infrared singularity only from the gluon propagators $F(l \pm r)$ in the down part of the graph. All other propagators have no divergences in this limit.

It can be shown that in the leading proportional to $s$ terms of the $\left\langle T_{a}\right\rangle$ and $\left\langle T_{b}\right\rangle$ amplitudes the integrals over $d^{2} k_{\perp}$ and $d^{2} l_{\perp}$ are factorized completely. They can be written in the form

$$
\left\langle T_{a, b}(s, t)\right\rangle=A^{2 g}(s, t) \hat{B}_{a, b},
$$

where

$$
A^{2 g}(s, t)=4 i s \alpha_{s}^{2} c_{2} \int d^{2} l_{\perp} \Pi(F(l \pm r)), \quad c_{2}=\frac{8}{36}
$$

is the born two-gluon high-energy amplitude, Fig.1. The magnitudes $F(l \pm r)$ are determined in (3). The $A^{2 g}$ amplitude has an infrared divergence in the $\lambda \rightarrow 0$ limit. The amplitudes $\hat{B}_{a, b}$ containing the integration over $d^{2} k_{\perp}$ are free from the singularities at $\lambda=0$. The factorization (4) can be proved for the $\left\langle T_{c}\right\rangle$ term on the basis of some approximations only and we use (4) as a definition for $B_{c}$

$$
\left\langle T_{c}(s, t)\right\rangle=A^{2 g}(s, t) \hat{B}_{c} .
$$


Let us define the following magnitude

$$
\hat{V}_{\mu \nu}(p, r)=\gamma_{\mu}(\not p+m) \gamma_{\nu}+\hat{B}_{\mu \nu}(p, r) .
$$

It is the imaginary part of the quark-two-gluon interaction with the exception of the $\delta$ function contributing to the high-energy amplitude (5). The graphical representation of $\hat{V}_{\mu \nu}$ is shown in figure 2. The first and second terms of $V_{\mu \nu}$ contain the upper parts of the born two-gluon amplitude and of the graphs, Fig.1, respectively. We can introduce now the function

$$
\hat{V}=\hat{V}_{\mu \nu}(p, r) \frac{p^{\prime \mu} p^{\prime \nu}}{s^{2}}
$$

Here, the structure $p^{\mu} p^{\prime \nu}$ is connected with the spin-non-flip matrix element in the down quark line.

It can be shown that the sum of the graphs, Fig.1,

$$
\left\langle T^{2 g}(s, t)\right\rangle=A^{2 g}(s, t)\left[\hat{\Gamma}_{0}+\sum_{i=a . . c} \hat{B}_{i}\right]
$$

can be written in the form

$$
\left\langle T^{2 g}(s, t)\right\rangle=A^{2 g}(s, t) \hat{V} .
$$

So we can conclude that $\hat{V}$ is a quark-pomeron vertex.

In (9) we use the definition

$$
\hat{\Gamma}_{0}=\frac{\not p^{\prime}}{s}
$$

The $\hat{\Gamma}_{0}$ structure is completely equivalent to the $\gamma_{\mu} \otimes \gamma^{\mu}$ matrix product that reflects a well-known fact that the spinless quark-pomeron coupling is like a $C=+1$ isoscalar photon (see [4] e.g).

The $\hat{B}_{i}$ in (9) can be decomposed over the independent matrix structures

$$
\hat{B}_{i}=\sum_{k=0}^{4} B_{i}^{k}(t) \hat{\Gamma}_{k}
$$

Here, the functions $B_{i}^{k}(t)$ have the terms independent of energy which we shall calculate in what follows. In addition to $\hat{\Gamma}_{0}$ we use here the following definitions:

$$
\hat{\Gamma}_{1}=I, \hat{\Gamma}_{2}=\not p, \hat{\Gamma}_{3}=\frac{i \epsilon^{\alpha \beta \gamma \rho} p_{\alpha} p_{\beta}^{\prime} r_{\gamma} \gamma_{\rho} \gamma_{5}}{s}, \hat{\Gamma}_{4}=\frac{i m \sigma^{\alpha \beta} p_{\alpha}^{\prime} r_{\beta}}{s} \text {. }
$$

As a result of such a normalization in $(11,13)$, we have no energy dependences in the spin-flip and non-flip matrix elements of $\left\langle\hat{\Gamma}_{i}\right\rangle$. The analyses of different matrix structure contributions to $\left\langle\hat{B}_{i}\right\rangle_{f l i p}$ can be found in [8].

Note that we must exclude from $\hat{B}$ in (9) the spin-non-flip part connected with the $\hat{\Gamma}_{0}$ contribution to avoid a double counting in summation of the pomeron graphs, as we shall see later. So, the born term in $\hat{V}$ (Fig.2) determines the spin-non-flip part $\sim \hat{\Gamma}_{0}$ and the upper box subgraphs from Fig.1 are the lower $\alpha_{s}$ order contribution 
to the spin-dependent part of this vertex. Really, calculating the spin-non-flip and spin-flip matrix elements in the upper quark line of the vertex function we find

$$
\begin{array}{r}
V(t)_{n o n-f l i p}=\langle\hat{V}\rangle_{\text {non-flip }}=\left(1+O\left(\alpha_{s}\right)\right) \\
V(t)_{f l i p}=\langle\hat{V}\rangle_{f l i p}=\sum_{i=a . . c}\left\langle\hat{B}_{i}\right\rangle_{f l i p} .
\end{array}
$$

These matrix elements are independent of energy.

The results of calculations [8] of $\left\langle\hat{B}_{i}\right\rangle_{\text {slip }}$ for the diagrams of Fig.1 are shown in Fig.3. In calculations we use $\lambda=.1 \mathrm{GeV}$ and $\alpha_{s}=.3$ which is typical of $\left|q^{2}\right| \simeq 1 \mathrm{GeV}^{2}$. The total spin flip amplitude $V(t)_{\text {flip }}$ is about 2 per cent of the $V(t)_{\text {non-flip. }}$.

Let us use as a model of the pomeron the set of ladder type gluon graphs in the leading logarithm approximation [10]. In this case, the $\hat{\Gamma}_{0}$ structure determines the leading terms in the spin-non-flip part of the scattering amplitude. Naturally, we can determine the quark-quark scattering amplitude for the pomeron ladder diagrams $A_{2 g}^{n}(s, t)$ in the $\alpha_{s}^{n}$ order with taking into account the quark-pomeron vertex as follows:

$$
\left\langle T_{n}(s, t)\right\rangle=A_{n}^{2 g}(s, t) \hat{V},
$$

that coincides for $n=2$ with the amplitude (10).

It is easy to see that we must really exclude the contribution of $\hat{\Gamma}_{0}$ term from the graph, Fig.1a, to the vertex function in $\left\langle T_{n}(s, t)\right\rangle$ because the same term can be found in $\left\langle T_{n+1}(s, t)\right\rangle$.

After summation of the gluon ladder diagrams we have

$$
\langle T(s, t)\rangle=\sum_{n=2}^{\infty} A_{n}^{2 g}(s, t) \hat{V}=P(s, t) \hat{V} .
$$

Here, $P(s, t)$ is a spinless pomeron containing the sum of gluon ladder graphs. Its calculations and summations have been performed in the leading logarithmic approximation (see [11] e.g).

It is easy to see that the form (16) leads to the same energy dependence in the spin-flip and non-flip amplitudes

$$
\frac{\left|T_{f l i p}(s, t)\right|}{\left|T_{\text {non-flip }}(s, t)\right|} \simeq V(t)_{f l i p}
$$

This quantity is of an order of $\alpha_{s}$. It is independent of energy in contrast with (1) but to obtain (1) we use the amplitudes of the same order in $\alpha_{s}$ unlike (16). Note that the summation only of the gluon ladder diagrams has been performed here. The complete investigation of this problem is very complicated.

So we see that the upper parts of the graphs, Fig.1a,b determine the lower in $\alpha_{s}$ contributions to the spin-flip part of the quark-pomeron vertex. All momenta in these subgraphs are about $p$ ( $p^{\prime}$ components are suppressed by $\left.s\right)$. As a result, these parts of the diagrams are at low energies because we cannot obtain any large magnitude $\sim s$ from the scalar products of the momenta.

Thus, we obtain the factorization of the amplitude into the high energy spinless pomeron $(P(s, t))$ and low energy spin-dependent vertex function $(\hat{V})$. We observed 
this effect in our previous model investigations [7]. It is shown that the gluons in the high energy ladder diagrams make the spin-flip amplitude growing as $s$. This means the existence of the spin-flip part in pomeron exchange. The perturbative calculations show that the ratio of the spin flip and non-flip amplitudes is not very large for the on-mass-shell quarks (only a few per cent) but the off-mass-shell effects can also increase the spin-flip contribution essentially [7]. Note that the quark-pomeron vertex function $\hat{V}$ is determined at small momenta transfer by the large-distance contributions. So for the realistic estimations of this magnitude it is necessary to take into consideration the nonperturbative effects.

Acknowledgement. The author would like to thank Professor Abdus Salam, the International Atomic Energy Agency and UNESCO and Professor A.Di.Giacomo, Dipartimento di Fisica dell'Universita'di Pisa, for hospitality at the International Centre for Theoretical Physics, Trieste and Instituto Nazionale di Fisica Nucleare, Sezione di Pisa where this work was completed. He expresses his deep gratitude to D.V.Shirkov, A.V.Efremov, V.A.Matveev, V.A.Meshcheryakov, M.Mintchev, R.Peschanski, O.V.Seljugin and O.V.Teryaev for fruitful discussions. 


\section{References}

[1] S.B. Nurushev, in: Proc. of the 2 Int. Workshop on High Energy Spin Phys. (Protvino, 1984), p. 5;

A.D.Krish, in: Proc. of the 6 Int. Symp. on High Energy Spin Phys. (Marseille,1984), p. C2-511;

N.E.Tyurin, in: Proc. of the 8 Int. Symp. on High Energy Spin Phys. (Bonn,1990), p. 65

[2] S.J.Brodsky, G.P.Lepage, Phys.Rev. D22 (1980) 2157

[3] F.E.Low, Phys.Rev. D12 (1975) 163;

S.Nussinov, Phys.Rev.Lett. 34 (1975) 1286

[4] P.V.Landshoff, O. Nachtmann, Z.Phys.C-Particles and Fields 35 (1987) 405

[5] D.A.Ross, J.Phys. G:Nucl.Part.Phys. 15 (1989) 1175

[6] S.V.Goloskokov, Yad.Fis. 49 (1989) 1427

[7] S.V.Goloskokov, J.Phys. G:Nucl.Part.Phys. 19 (1993) 67

[8] S.V.Goloskokov, O.V.Selyugin, JINR Prepr. E2-93-164, Dubna (1993)

[9] B.M.McCoy, T.T.Wu, Phys.Rev. D12 (1975) 3257;

H.T.Nich, Y.P.Yao, Phys.Rev. D13 (1976) 1082;

L.Tybursky, Phys.Rev. D13 (1976) 1107

[10] L.V.Gribov, E.M.Levin, M.G.Ryskyn, Phys.Rep. 100 (1983) 151

[11] E.A.Kuraev, L.N.Lipatov, S.V.Fadin, Zh.Eksp.Teor.Fiz. 72 (1977) 377 


\section{FIGURE CAPTIONS}

Fig.1 The born two-gluon $q q$ diagram and the $\alpha_{s}^{3}$ contributions to the spin-flip amplitude.

Fig.2 The contributions to $\hat{V}_{\mu \nu}$ from the upper parts of the born two-gluon graph and of the diagrams, Fig.1a-c.

Fig.3 The spin-flip amplitudes $\left\langle\hat{B}_{a-c}(t)\right\rangle_{f l i p}$; full curve - sum of the amplitudes. 


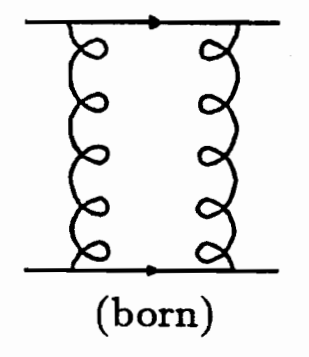

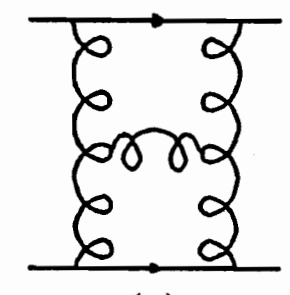

(a)

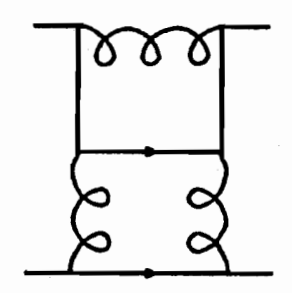

(b)

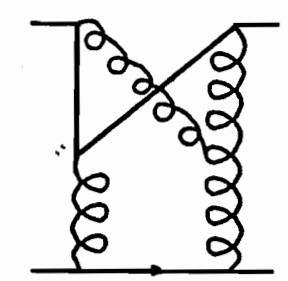

(c)

Fig.1

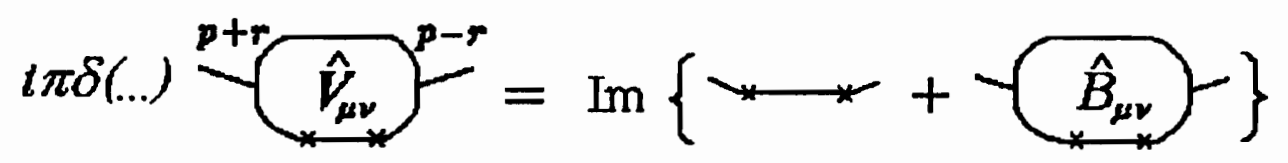

Fig.2

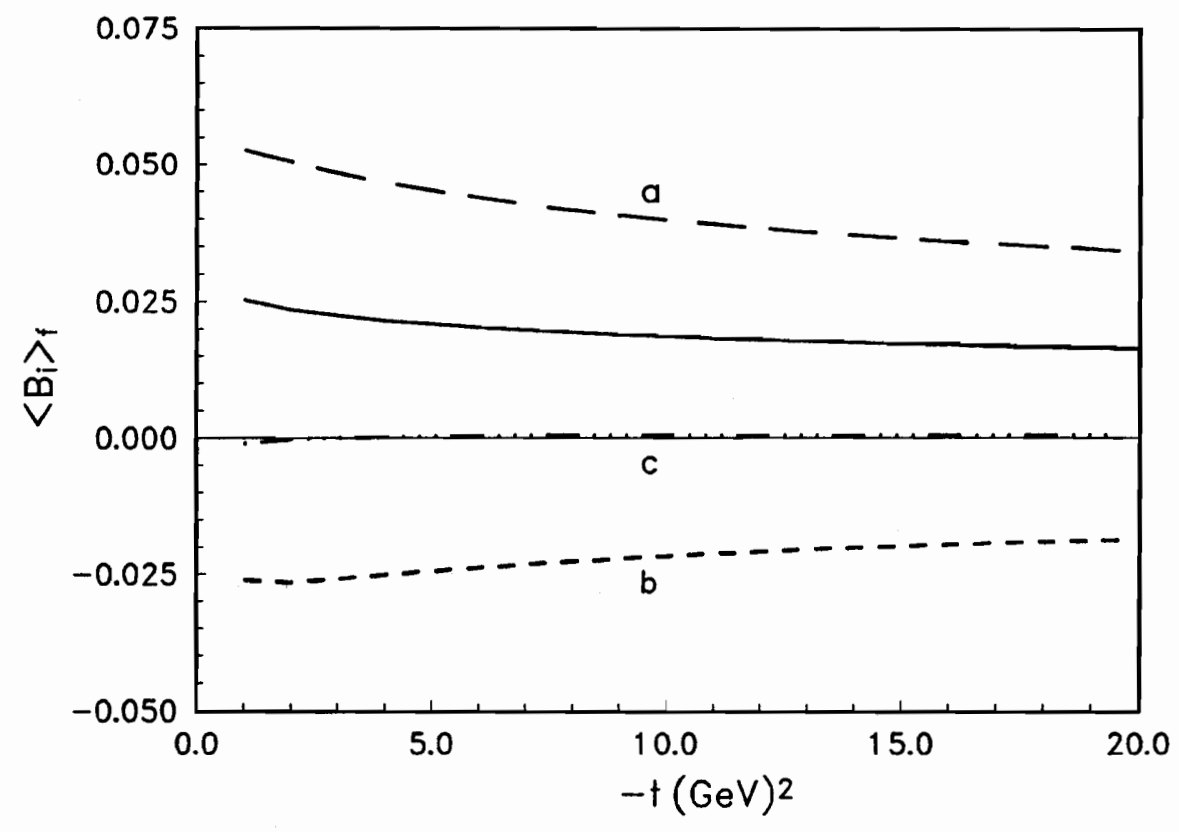

Fig.3 